\title{
WHAT ELSE about...Hypoellipticity?
}

\section{Brian Street}

\author{
Communicated by Cesar E. Silva
}

EDITOR'S NOTE. This is a deeper follow-up to "WHAT IS...Hypoellipticity?" on p. 418.

Let $\mathcal{P}=\sum_{|\alpha| \leq m} a_{\alpha}(x) \partial_{x}^{\alpha}$ be a linear partial differential operator of degree $m$ on $\mathbb{R}^{n}$ with smooth coefficients $a_{\alpha} \in C^{\infty}\left(\mathbb{R}^{n}\right)$. Consider the equation $\mathcal{P} u=f$ (where $u$ and $f$ are distributions). A basic problem is: given $f$ what can one say about $u$ ? Even the simplest $\mathcal{P}$ have nontrivial nullspaces, so one cannot hope to recover $u$ completely from $f$. One often resorts to understanding what sort of properties $u$ inherits from $f$. For example, if $f$ is smooth, does it follow that $u$ is smooth?

Definition. We say $\mathcal{P}$ is hypoelliptic at $x_{0} \in \mathbb{R}^{n}$, if whenever $\mathcal{P} u$ is $C^{\infty}$ on a neighborhood of $x_{0}$, then $u$ is $C^{\infty}$ on a neighborhood of $x_{0}$.

Example 1. On $\mathbb{R}, \mathcal{P}=\frac{d}{d x}$ is hypoelliptic at every point $x_{0} \in \mathbb{R}$. This follows from the fundamental theorem of calculus: $u(x)=\int_{X_{0}}^{x} \mathcal{P} u(t) d t+C$.

Example 2. On $\mathbb{R}^{2}$ with coordinates $(x, y), \mathcal{P}=\frac{\partial}{\partial x}$ is not hypoelliptic at any $\left(x_{0}, y_{0}\right) \in \mathbb{R}^{2}$. Indeed $\mathcal{P} u=0$ for any $u$ that depends only on $y$, and thus $u$ need not be smooth.

We can formulate a more quantitative version of hypoellipticity using Sobolev spaces. For $p \in[1, \infty]$, $s \in \mathbb{R}$, let $L_{s}^{p}$ denote the $L^{p}$ Sobolev space of order $s$ on $\mathbb{R}^{n}$ (sometimes denoted by $W^{s, p}$ ). When $s \in \mathbb{N}$, this is the Banach space of distributions $v$ such that $\partial^{\alpha} v \in L^{p}$, $|\alpha| \leq s$. We also use the following notation. For two

Brian Street is associate professor of mathematics at the University of Wisconsin-Madison. His email address is street@math .wisc. edu.

For permission to reprint this article, please contact: reprint-permission@ams.org.

DOI: http://dx.doi.org/10.1090/noti1664 functions $\phi_{1}, \phi_{2} \in C_{0}^{\infty}\left(\mathbb{R}^{n}\right)$, we write $\phi_{1} \prec \phi_{2}$ if $\phi_{2} \equiv 1$ on a neighborhood of the support of $\phi_{1}$. Furthermore, we write $\left\{x_{0}\right\} \prec \phi_{1}$ if $\phi_{1} \equiv 1$ in a neighborhood of $x_{0}$.

A simple consequence of the Sobolev embedding theorem is that $v$ is $C^{\infty}$ near $x_{0} \in \mathbb{R}^{n}$ if and only if there exists $\left\{x_{0}\right\} \prec \phi \in C_{0}^{\infty}$ such that $\phi v \in \bigcap_{s} L_{S}^{2}$. From this the next lemma is immediate.

Lemma 1. Let $\left\{x_{0}\right\} \prec \phi_{1} \prec \phi_{2}$. Suppose $\forall s \in \mathbb{R}, \exists r(s) \in$ $\mathbb{R}$ such that $\phi_{2} \mathcal{P} u \in L_{r(s)}^{2} \Rightarrow \phi_{1} u \in L_{s}^{2}$. Then $\mathcal{P}$ is hypoelliptic at $x_{0}$.

One of the most classical hypoelliptic operators is the Laplacian on $\mathbb{R}^{n}: \triangle=-\sum_{j=1}^{n} \partial_{x_{j}}^{2}$. For $\Delta u=f$, taking the Fourier transform yields $|\xi|^{2} \hat{u}=\hat{f}$. Thus, one can try to solve for $u$ by taking the inverse Fourier transform of $|\xi|^{-2} \hat{f}$. There are several difficulties in making this argument precise; once they are overcome, this idea can be used to show that $\Delta$ is hypoelliptic at every point in $\mathbb{R}^{n}$.

Remarkably, the same idea can be used for a wide class of operators that do not have constant coefficients: the elliptic operators. This is one of the most basic applications of pseudodifferential operators, which were introduced by Kohn and Nirenberg and whose properties were studied and applied by Hörmander and many others.

Definition. We say that $\mathcal{P}$ is elliptic at $x_{0}$ if

$$
\sum_{|\alpha|=m} a_{\alpha}\left(x_{0}\right) \xi^{\alpha}=0
$$

only if $\xi=0$.

Let $\mathcal{P}$ be elliptic at $x_{0}$ and fix $\{0\} \prec \psi$. For $x$ near $x_{0}$, the inverse Fourier transform in $\xi$ (treating $x$ as a constant) of $(1-\psi(\xi))\left(\sum_{|\alpha|=m} a_{\alpha}(x) \xi^{\alpha}\right)^{-1} \hat{f}(\xi)$ is a function of $x$ alone; let $T f(x)$ denote this function. Then, $T \mathcal{P} u=u+e$, where $e$ is smoother than $\mathcal{P} u$. Iterating this result leads to the hypoellipticity of $\mathcal{P}$ at $x_{0}$. In fact, one can show that there 
exist $\left\{x_{0}\right\} \prec \phi_{1} \prec \phi_{2}$ such that for $p \in(1, \infty), s \in \mathbb{R}$, $\phi_{2} \mathcal{P} u \in L_{s-m}^{p} \Rightarrow \phi_{1} u \in L_{s}^{p}$. Thus $u$ is smoother than $\mathcal{P} u$ by $m$ derivatives. Since $\mathcal{P}$ is a differential operator of order $m$, this is the best one could possibly hope for. See [3] for further details and a history of these ideas.

Hypoellipticity becomes more subtle when $\mathcal{P}$ is not elliptic.

Definition. We say $\mathcal{P}$ is subelliptic at $x_{0}$ if $\exists \epsilon>0$ such that the conditions of Lemma 1 hold with $r(s)=s-\epsilon$.

Thus if $\mathcal{P}$ is subelliptic at $x_{0}$, then $u$ is smoother than $\mathcal{P} u$ by $\epsilon$ derivatives (in $L^{2}$ Sobolev spaces). This can be much weaker than ellipticity, where we have $\epsilon=m$. It is clear from Lemma 1 that if $\mathcal{P}$ is subelliptic at $x_{0}$, then $\mathcal{P}$ is hypoelliptic at $x_{0}$. Unlike for elliptic operators, recognizing if an operator is subelliptic can be quite hard.

Let $X_{1}, \ldots, X_{r}$ be smooth vector fields on $\mathbb{R}^{n}$. Define $\mathcal{L}=X_{1}^{*} X_{1}+\cdots+X_{r}^{*} X_{r}$, where $X_{j}^{*}$ denotes the formal $L^{2}$ adjoint of $X_{j}$. (When $X_{j}=\frac{\partial}{\partial x_{j}}, j=1, \ldots, n$; then $\mathcal{L}=\triangle$.)

Example 3. If the vector fields $X_{1}, \ldots, X_{r}$ are tangent to a lower dimensional submanifold of $\mathbb{R}^{n}$ passing through $x_{0}$, then $\mathcal{L}$ is not hypoelliptic at $x_{0}$. This generalizes Example 2.

Hörmander dealt with the opposite situation.

Definition. We say $X_{1}, \ldots, X_{r}$ satisfy Hörmander's condition at $x_{0}$ if the Lie algebra generated by $X_{1}, \ldots, X_{r}$ spans the tangent space at $x_{0}$.

Hörmander proved that if $X_{1}, \ldots, X_{r}$ satisfy this condition at $x_{0}$, then $\mathcal{L}$ is subelliptic at $x_{0} \cdot \mathcal{L}$ is called the Hörmander sub-Laplacian.

Example 4. On $\mathbb{R}^{2}$, let $X_{1}=\frac{\partial}{\partial x}, X_{2}=x \frac{\partial}{\partial y}$. Then $\left[X_{1}, X_{2}\right]=$ $\frac{\partial}{\partial y}$ so that $X_{1}, X_{2}$ satisfy Hörmander's condition at every $\left(x_{0}, y_{0}\right) \in \mathbb{R}^{2}$. Thus, $\mathcal{L}=X_{1}^{*} X_{1}+X_{2}^{*} X_{2}=-\partial_{x}^{2}-x^{2} \partial_{y}^{2}$ is subelliptic at every $\left(x_{0}, y_{0}\right) \in \mathbb{R}^{2} . \mathcal{L}$ is not elliptic at any point of the form $\left(0, y_{0}\right)$.

In fact, Hörmander's sub-Laplacian is one of the most fundamental examples of a far-reaching generalization of ellipticity, known as maximal hypoellipticity. Let $Q(y)=\sum_{|\alpha| \leq m} b_{\alpha}(x) y^{\alpha}$ be a polynomial of degree $m$ in noncommuting indeterminates $y_{1}, \ldots, y_{r}$, with smooth coefficients $b_{\alpha} \in C^{\infty}\left(\mathbb{R}^{n}\right)$ : here we have used ordered multi-index notation to deal with the noncommuting indeterminates. If $X_{1}, \ldots, X_{r}$ are vector fields, then it makes sense to consider $\mathcal{P}=Q\left(X_{1}, \ldots, X_{r}\right)$ as a partial differential operator of degree at most $m$.

Definition. Suppose $X_{1}, \ldots, X_{r}$ satisfy Hörmander's condition at $x_{0}$. We say $\mathcal{P}=Q\left(X_{1}, \ldots, X_{r}\right)$ is maximally hypoelliptic at $x_{0}$ if there exist $\left\{x_{0}\right\} \prec \phi_{1} \prec \phi_{2}$ such that $\phi_{2} \mathcal{P} u \in L^{2} \Rightarrow \phi_{1} X^{\alpha} u \in L^{2}$ for all ordered multi-indicies $\alpha$ with $|\alpha| \leq m$.
A general method of a priori estimates developed by Kohn can be used to show that maximally hypoelliptic operators are subelliptic. Beyond subellipticity, Kohn's method does not give a complete understanding of maximal hypoellipticity, and unlike the case of elliptic operators, the Fourier transform is not a decisive tool. Despite these problems, many of the classical results for ellipticity generalize to the case of maximal hypoellipticity using singular integral operators. This was carried out by many authors, led by E. M. Stein. See [2, Ch. 2] for details and a history of these ideas.

Unfortunately, it can be quite difficult to recognize when a particular operator is maximally hypoelliptic. There is a deep conjecture of Helffer and Nourrigat relating this to representations of nilpotent Lie groups; however this conjecture remains open.

Finally, we mention a delicate phenomenon which is far from being understood: hypoelliptic operators that are not subelliptic. For example, Kohn considered the setting of Hörmander's sub-Laplacian, but with real vector fields replaced by complex vector fields [1]. On $\mathbb{R}^{2}$, let $L=\partial_{x}+i x \partial_{y}, \bar{L}=\partial_{x}-i x \partial_{y}$. Set $\mathcal{L}=\bar{L}^{*} \bar{L}+\left(x^{k} L\right)^{*} x^{k} L$; it is easy to see that the complex Lie algebra generated by $\bar{L}$ and $x^{k} L$ spans the complexified tangent space at every point (as in Hörmander's condition). However, Kohn showed that $\mathcal{L}$ is not subelliptic for $k \geq 1$ : at the point $(0,0), \mathcal{L}$ satisfies the conditions of Lemma 1 with $r(s)=s+k-1$, and no better. Hence, counterintuitively, $\mathcal{L} u$ might be more smooth than $u$, despite the fact that $\mathcal{L}$ is hypoelliptic. Surprisingly, it is a result of Christ that in $\mathbb{R}^{3}$ with coordinates $(x, y, t)$, the operator $\mathcal{L}+\partial_{t}^{*} \partial_{t}$ is not hypoelliptic at $(0,0,0)$. Thus the complex analog of Hörmander sub-Laplacians might be subelliptic, might be hypoelliptic but not subelliptic, or might not be hypoelliptic at all. Besides these and some other intriguing results, many aspects of hypoellipticity without subellipticity remain uncharted territory.

In conclusion, we have: Ellipticity $\Rightarrow$ Maximal Hypoellipticity $\Rightarrow$ Subellipticity $\Rightarrow$ Hypoellipticity, and none of the reverse implications hold.

\section{References}

[1] J. J. KoHN, Hypoellipticity and loss of derivatives, Ann. of Math. (2) 162 (2005), no. 2, 943-986. MR2183286

[2] BRIAN STREET, Multi-parameter Singular Integrals, Ann. of Math. Stud., vol. 189, Princeton University Press, Princeton, NJ, 2014. MR3241740

[3] FrançOIS TRÈVES, Introduction to Pseudodifferential and Fourier Integral Operators, Vol. 1, Plenum Press, New York-London, 1980. MR597144

\section{ABOUT THE AUTHOR}

Brian Street's area of research is harmonic analysis and singular integrals. In his spare time, he enjoys reading and biking. 